\title{
Conservación de los Bosques Relictos del NO de Perú
}

El presente documento resume las conclusiones obtenidas en el «Taller sobre Bosques Relictos de las Vertientes Occidentales Andinas del Norte del Perú y Sur del Ecuador», desarrollado en el marco del X Congreso Nacional de Botánica, Trujillo-Perú, el 04 de mayo del 2004. En este taller se llegaron a las siguientes conclusiones, dirigidas principalmente a los gobiernos regionales y concejos provinciales que incluyen a los departamentos de Cajamarca, Lambayeque, La Libertad y Piura:

-El Perú esta reconocido entre los países tropicales con mayor diversidad vegetal. La región norte del país incluye un porcentaje alto de esa diversidad. Entre los ecosistemas importantes que concentra la diversidad en esta región se encuentran los bosques relictos de la vertiente occidental de los Andes (Piura, Lambayeque, Cajamarca, La Libertad), considerados unos de los más altamente endémicos en todo el mundo. Estos bosques que se extienden hasta los $12^{\circ} \mathrm{S}$, tienen tanto una diversidad muy elevada como un gran índice de endemismo. Muchas de estas especies están restringidas a bosques relictos individuales, tales como en los siguientes departamentos:

\section{LA LIBERTAD \\ Prov. Gran Chimú}

Bosque de Lucma

\section{CAJAMARCA}

\section{Prov. San Miguel de Pallaques}

Bosque de Tongod, Bosques de Santa RosaEl Palmo, Bosque del Cerro Quillón, Bosque de La Oscurana, Niepos y Corral Viejo

\section{Prov. San Miguel y Santa Cruz}

Bosques Los Cedros y Cascarilla

\section{Prov. Santa Cruz}

Bosque de Monteseco (incl. La Florida, Taulis)

\section{Prov. Contumazá}

Bosque de San Mateo o Cachil

\section{Prov. Cutervo}

Bosques de Querocotillo-Granadillo-Shinshin Sur (río Chotano), Bosques de Cutervo

\section{LAMBAYEQUE \\ Prov. Ferreñafe}

Bosques húmedos de Kañaris (Upaipeta), Bosques de Chiñama- Mamahuaca-Bosque del Cerro Pluto (Santa Lucía Kañaris), Bosques de Yatrapa

\section{PIURA}

\section{Prov. Huancabamba}

Bosque de Canchaque

\section{Prov. Ayabaca}

Bosques de Ayabaca (Huamba, El Toldo, Aypate, Cuyas), Bosque de Mijal

- A pesar de su gran importancia biológica y ecológica, estos bosques siguen en peligro de destrucción. Alrededor del 80\% de su área está destruida en la actualidad, básicamente por la actividad antrópica y el resto está muy intervenido. Estos ambientes son muy importantes como para permitir que sean exterminados.

Respecto a la situación actual de estos bosques, en el taller se llegó a las siguientes conclusiones:

Los bosques relictos son los ecosistemas en mayor peligro de extinción total en el Perú.

Es predecible que la destrucción de estos ecosistemas afectará el ciclo natural hídrico, disminuyendo el caudal de los ríos, afectando a las poblaciones que dependen de sus aguas y por extensión a la agricultura.

Algunos de estos bosques relictos tienen un alto potencial para el ecoturismo, a través de su belleza escénica y paisajística, junto con su riqueza biológica.

Es necesaria más investigaciones, especialmente en los bosques menos explorados, como los bosques de Kañaris, Mijal, Lucma, Bolívar, Querocotillo y Quillón. Es un deber para la comunidad científica nacional e internacional, realizar estudios de parámetros físicos y condiciones biológicas explicando la composición, riqueza y endemismo. 
La investigación debería enfocarse tanto en áreas de taxonomía biológica (incluyendo florística y revisiones taxonómicas detalladas), así como en las áreas de hidrología, ecología y fitosociología.

Por su alto valor ecológico y económico la preservación de estos ecosistemas, a través de la instalación de zonas intangibles, reservas regionales, etc., debe tener alta prioridad para las autoridades regionales y urgentemente necesita su atención.

La conservación de estos bosques debe basarse en una política de desarrollo sostenible, procurando fuentes alternativas para los recursos actualmente extraídos del bosque por la población.

En especial se recomienda la reforestación de las márgenes de los bosques con especies nativas de alto valor económico tales como aliso, cedro, lúcuma del oso, olivo o saucesillo, con la participación total de la población afectada por la creación de zonas protegidas.

Merecen una especial atención los bosques más grandes y biológicamente más ricos que todavía quedan y que se encuentran en un peligro inmediato de destrucción, especialmente los bosques de Monte Seco, Kañaris, Lucma, cerro Quillón, Los Cedros y Cascarilla, Ayabaca, Huambo y La Oscurana.

\section{Lista de adherentes}

1. Leopoldo Vásquez Núñez El Cabildo 162, Urb.Latina, Chiclayo.

2. Manuel Charcape Ravelo manuelbot@hotmail.com

3. Mario López Mesones lopezmesones@yahoo.es

4. Edgard E. Vicuña Miñano edgardobotanico23@hotmail.com

5. Fany Palomino Zeña refringente@hotmail.com

6. Lisset Tonder González Bioli5ina@hotmail.com

\section{Lima, 8 de diciembre 2005}

\section{Maximilian Weigend}

Institut für Biologie - Systematische Botanik und Pflanzengeographie, Freie Universität Berlin

E-mail: weigend@zedat.fu-berlin.de
7. Lizzethe Bellido Huertas Lizzethe1301@hotmail.com

8. Shirley Judith Guerrero Muñoz shirley_judith20@hotmail.com

9. Fabiola A. Parra Rondinel quisuar@yahoo.es

10. Joaquin Ricardo Martos Ugaz jorimau@hotmail.com

11. Mariella Scarpati Gavino mazulizo@hotmail.com

12. Catherine Bravo Ávila wankartipa@fastmail.fin

13. Manuel E. Sotomayor V. Manuelernestosv@yahoo.es

14. Ana María Juárez Chunga juarezii@hotmail.com

15. Susy Castillo Ramón susy-827@hotmail.com

16. Luis Vargas Avilés luisalnus@yahoo.es

17. Melissa Quispe Gonzales melissa_giuliana@hotmail.com

18. Munirin Damian Peralta mdamper@hotmail.com

19. Dina Balarezo Cabrejos dinabalarezo@hotmail.com

20. Duberti Elera Gonzáles duberelera@hotmail.com

21. Ayasta Varona José ayastae@hotmail.com

22. Roxana Aguirre Tocas roxanat14@hotmail.com

23. John Vargas Martínez excalibur333@hotmail.com

24. Erixs Montoya Guzmán erixs98@hotmail.com

25. Carlos Loudeo Béjar matlewlouder632@hotmail.com

26. Nelly Melgarejo Salas neli320@hotmail.com

27. Miriam Sosa Chiroque misochi@hotmail.com

28. Sandra Rodríguez Rodríguez sandrarodríguez16@hotmail.com

29. Rimarachín Cayotopa Leyda G. gueiler90@hotmail.com

30. Medina Ibáñez Víctor medibiol@hotmail.com

31. César Arana Bustamante caranab@unmsm.edu.pe

32. Sandra Arroyo Alfaro sandrarroyoa@yahoo.com

\section{Eric F. Rodríguez Rodríguez}

Herbarium Truxillense (HUT), Universidad Nacional de Trujillo, Trujillo-Perú

E-mail: efrr@unitru.edu.pe 